\title{
Mycobacterium noviomagense sp. nov.; clinical relevance evaluated in 17 patients
}

\author{
Jakko van Ingen, ${ }^{1,2}$ Martin J. Boeree, ${ }^{1}$ Wiel C. M. de Lange, ${ }^{1}$ \\ Petra E. W. de Haas, ${ }^{2}$ Adri G. M. van der Zanden, ${ }^{3}$ Wouter Mijs, ${ }^{4}$ \\ Leen Rigouts, ${ }^{5}$ P. N. Richard Dekhuijzen ${ }^{1}$ and Dick van Soolingen ${ }^{2}$ \\ ${ }^{1}$ Radboud University Nijmegen Medical Center, Department of Pulmonary Diseases, Nijmegen, \\ The Netherlands \\ ${ }^{2}$ National Institute for Public Health and the Environment, National Mycobacteria Reference \\ Laboratory, Bilthoven, The Netherlands \\ ${ }^{3}$ Laboratory for Medical Microbiology and Public Health, Enschede, The Netherlands \\ ${ }^{4}$ Innogenetics N.V., Department of Diagnostics, Gent, Belgium \\ ${ }^{5}$ Institute of Tropical Medicine, Department of Mycobacteriology, Antwerp, Belgium
}

Correspondence Jakko van Ingen jakko.van.ingen@rivm.nl
Improved detection and identification techniques have triggered renewed interest in non-tuberculous mycobacteria (NTM) and their role as opportunistic pathogens. PCR techniques and 16S rRNA gene sequence analysis have brought to light a series of novel NTM species, however, the clinical relevance of these species is not always clear (Tortoli, 2003; Tortoli et al., 2001; Griffith et al., 2007).

NTM infections present predominantly as chronic pulmonary disease, although extrapulmonary and disseminated infections have also been described (Griffith et al., 2007). Local immunosuppression due to pre-existing pulmonary disease and systemic immunosuppression, e.g. in haema-

\footnotetext{
Abbreviations: ATS, American Thoracic Society; ITS, internal transcribed spacer; NTM, non-tuberculous mycobacteria.

The GenBank/EMBL/DDBJ accession numbers 16S rRNA, 16S-23S ITS 1 and 2, hsp65 and rpoB gene sequences of strain NLA000500338 ${ }^{\top}$ are EU239955, EU439248, EU439249, EU600390 and EU810775, respectively

Phylogenetic trees based on $h s p 65$ and rpoB gene sequences of selected mycobacterial species are available as supplementary material with the online version of this paper.
}

tological malignancy, immunosuppressive medication and HIV/AIDS, have been identified as predisposing factors (Griffith et al., 2007) for NTM infections. Infection has to be differentiated from contamination and pseudo-infection, characterized by the recovery of single NTM isolates from the respiratory or digestive tract without signs of disease (Griffith et al., 2007; Portaels, 1995). Their ubiquitous presence in the environment, survival in flowing water systems and resistance to disinfectants implies that NTM often represent laboratory or medical equipment contamination (Griffith et al., 2007; Portaels, 1995; van Klingeren \& Pullen, 1993). The diagnostic criteria proposed in a Statement by the American Thoracic Society (ATS) are designed to differentiate between true infection and pseudo-infection or contamination, based on clinical, radiological and microbiological features (Griffith et al., 2007).

This study describes the grouping of 18 previously unknown Mycobacterium isolates with identical $16 \mathrm{~S}$ rRNA gene sequences and with a high degree of gene sequence similarity to strains of Mycobacterium xenopi. As other features of these novel strains were highly distinct 
from $M$. xenopi and the clinical relevance differed significantly between the new isolates and M. xenopi, the 18 isolates are proposed to represent a novel species of the genus Mycobacterium.

The 18 novel isolates were acquired from pulmonary samples (13 from sputum, 4 from broncho-alveolar lavage fluid and 1 from a post-mortem lung biopsy) of 17 patients in the Netherlands between January 1999 and January 2007. To determine clinical relevance, we examined the medical records of all 17 patients; their baseline characteristics are displayed in Table 1. The predominance of male patients, mean age and history of chronic pulmonary disease are comparable with previous NTM studies (Griffith et al., 2007; Henry et al., 2004). None of the patients had clinical and radiographic features suggestive of mycobacterial lung disease; one was systemically immunocompromised due to HIV co-infection. The post-mortem lung biopsy sample showed histological features of bronchopneumonia and invading bacteria, without features of mycobacterial disease such as granuloma formation. All patient samples were negative for acid-fast bacilli on direct microscopy which suggested the presence of a low number of NTM in the original sample or contamination after sample division for culture and microscopy. Although follow-up sputum cultures were performed for 16 patients, only one patient produced another culture that was positive for the novel strains. All others had a single positive culture. Based on these findings, none of the patients from whom the novel strains were isolated met the ATS criteria for pulmonary NTM disease (Griffith et al., 2007). Good clinical response to non-antimycobacterial regimes, the subsequent establishment of alternative diagnoses and the observed spontaneous conversion to negative cultures suggested that the novel strains were not the causative agent of these patients' symptoms. The novel

Table 1. Characteristics of the patients in the study group

The total number of patients was 17. COPD, Chronic obstructive pulmonary disease.

\begin{tabular}{|lc|}
\hline Characteristic & No. of patients \\
\hline Male & $13(77 \%)$ \\
Mean age (yr) (range) & $53(29-86)$ \\
Dutch origin & $16(94 \%)$ \\
Pre-existing pulmonary disease & $15(88 \%)$ \\
COPD & $8(47 \%)$ \\
Lung cancer & $4(24 \%)$ \\
Healed tuberculosis & $2(12 \%)$ \\
Recurrent pulmonary infection & $3(18 \%)$ \\
Bronchiectasis & $2(12 \%)$ \\
Current smoker & $5(29 \%)$ \\
Past smoker & $3(18 \%)$ \\
Alcohol abuse & $3(18 \%)$ \\
HIV infection & $1(6 \%)$ \\
\hline
\end{tabular}

*>3 In 6 months prior to sampling. strains therefore seem to have no clinical relevance, which distinguishes them from phylogenetically related, but more pathogenic species such as M. xenopi and Mycobacterium heckeshornense (Griffith et al., 2007; Roth et al., 2000; van Ingen et al., 2008).

Two patients received antituberculosis treatment for an average period of 10 weeks, after a presumptive diagnosis of TB. Treatment of patients not meeting the ATS diagnostic criteria is potentially harmful to patients in terms of adverse effects and costs (van Crevel et al., 2001).

Geographical clustering of the patients was observed, favouring the south-east of the Netherlands, in adjacent areas of the Limburg (7 cases), Gelderland (5 cases) and Noord-Brabant (2 cases) provinces. This clustering suggested the presence of the novel strains in specific local environments or tap water. Since the clustering was seen geographically, but not over time, contamination from medical machinery or the laboratories involved seemed less likely.

All of the novel isolates were subjected to laboratory diagnosis by the National Mycobacteria Reference Laboratory of the National Institute for Public Health and the Environment (RIVM). The RIVM provides molecular identification, drug susceptibility testing and epidemiological typing of mycobacterial isolates for all healthcare institutions in the Netherlands.

Biochemical identification and HPLC analysis of cell-wall mycolic acid content were performed using previously described approaches (Lévy-Frébault \& Portaels, 1992; Centers for Disease Control and Prevention, 1996). Eight isolates ( 4 M. xenopi, 4 of the novel strains) were subjected to biochemical testing. The results are detailed in Table 2. Morphologically, two patterns were clearly discernible. On Middlebrook 7H10 media, the M. xenopi isolates were scotochromogenic, showing yellow pigmentation, whereas

Table 2. Biochemical identification results for the novel isolates and closely related species

Taxa: 1, M. noviomagense sp. nov.; 2, M. xenopi; 3, M. heckeshornense (data from Roth et al., 2000); 4, M. botniense (Torkko et al., 2000). None of the taxa were able to tolerate $5 \% \mathrm{NaCl}$ in Lowenstein-Jensen (LJ) medium. +, Positive; -, negative; v, variable; NC, nonchromogenic; SC, scotochromogenic; NP, not published.

\begin{tabular}{|lcccc|}
\hline Characteristic & $\mathbf{1}$ & $\mathbf{2}$ & $\mathbf{3}$ & $\mathbf{4}$ \\
\hline Growth at $45{ }^{\circ} \mathrm{C}$ & - & + & + & + \\
Morphology & $\mathrm{NC}$ & $\mathrm{SC}$ & $\mathrm{SC}$ & $\mathrm{SC}$ \\
Colony size & Small & Large & $\mathrm{NP}$ & Small \\
Tolerance to (in LJ medium): & & & & \\
$\quad$ Isoniazid $10 \mu \mathrm{g} \mathrm{ml}^{-1}$ & - & - & - & $\mathrm{NP}$ \\
$\quad$ Thiophene 2-carboxylic acid & + & + & $\mathrm{NP}$ & $\mathrm{NP}$ \\
$\quad$ Hydroxylamine $250 \mu \mathrm{ml}^{-1}$ & $\mathrm{~V}$ & + & $\mathrm{NP}$ & $\mathrm{NP}$ \\
$\quad$ Para-nitrobenzoate $500 \mu \mathrm{g} \mathrm{ml}^{-1}$ & + & $\mathrm{V}$ & $\mathrm{NP}$ & $\mathrm{NP}$ \\
\hline
\end{tabular}


colonies of the novel species were smaller and nonchromogenic. All M. xenopi isolates grew at $45^{\circ} \mathrm{C}$, as previously reported (Torkko et al., 2000), but the novel strains were unable to grow at this temperature (Table 2). Biochemically, the novel isolates were indistinguishable from the cluster comprising M. xenopi, Mycobacterium botniense and $M$. heckeshornense, with negative results for urease, Tween 80 hydrolysis, niacin production, nitrate reductase, acid phosphatase and semi-quantitative catalase. HPLC of the novel isolates revealed a pattern characterized by one early and one late cluster of peaks, a profile similar to that of $M$. xenopi, M. heckeshornense and M. botniense (Fig. 1). The HPLC mycobacterium library (available online at http:// www.MycobacToscana.it) was used for this comparison.

Susceptibility testing was performed for eleven of the novel isolates from eleven patients using the agar dilution method (van Klingeren et al., 2007). For the novel isolates, we recorded in vitro resistance to rifampicin (MIC $2 \mathrm{mg} \mathrm{l}^{-1}$ ), resistance or intermediate susceptibility to ethambutol (MIC 10-20 $\mathrm{mg} \mathrm{l}^{-1}$ ) and intermediate susceptibility to isoniazid (MIC $0.5-1 \mathrm{mg} \mathrm{l}^{-1}$ ). The novel species proved susceptible in vitro to streptomycin, cycloserine, prothionamide, amikacin, ciprofloxacin, clofazimine, clarithromycin and rifabutin. The drug susceptibility pattern for the novel isolates differed slightly from clinical isolates of M. xenopi tested at RIVM which were mostly susceptible, in vitro, to rifampicin (MIC 0.5$1 \mathrm{mg} \mathrm{l}^{-1}$ ). These results for $M$. xenopi are in accordance with previous reports (Dauendorffer et al., 2002).

For molecular identification, sequencing of the full $16 \mathrm{~S}$ rRNA gene and 16S-23S internal transcribed spacer (ITS), partial rpoB and $h s p 65$ genes, and PCR restriction enzyme pattern analysis (PRA) of the $h s p 65$ gene were performed using previously described methods (Kim et al., 1999; Roth et al., 1998; Springer et al., 1996; Telenti et al., 1993). The sequences obtained were compared with the GenBank/EMBL (NCBI, http://www.ncbi.nlm.nih.gov) gene sequence databases.

The full $16 \mathrm{~S}$ rRNA gene sequence for the novel species showed $96 \%$ sequence similarity with that of M. xenopi and was $97 \%$ similar to those of M. heckeshornense and Mycobacterium shimoidei. A sequence difference of $1 \%$ has been proposed in the literature as the threshold for the designation of a novel species (Hall et al., 2003; Tortoli, 2003).

The full 16S rRNA gene sequence of the novel strains was aligned with those of reference strains of the closest related mycobacteria using CLUSTAL_X software (Thompson et al., 1997). The resulting topology and tree, inferred by neighbour-joining and visualized using the LOFT software package (van der Heijden et al., 2007) were evaluated by bootstrap analyses based on 1000 resamplings (Fig. 2).

Analysing only the hypervariable region A of the $16 \mathrm{~S}$ rRNA gene (151 bp), we found a $100 \%$ match in the GenBank/ EMBL database to a strain designated 'most closely resembling M. xenopi', as reported by Hall et al. (2003). Analysis of the phenotypic and genetic characteristics of the novel species (Table 2, Figs 1, 2 and 3) demonstrates that very subtle $16 \mathrm{~S}$ rRNA gene sequence differences can be associated with extensive divergence from the closest related species. This brings into question the use of monophasic identification methods.

Analysis of the 16S-23S ITS region revealed mixed sequence patterns for all 18 novel isolates, even from single colony cultures, and necessitated cloning. For cloning of the ITS, amplicons were generated using primers provided with the INNO LiPA Mycobacteria v2 kit (Innogenetics), and cloned in the PGEM-T Easy vector (Promega) according to the manufacturer's instructions. Thirty colonies of transformed Escherichia coli strain DH5F were picked for each sample, cultured and used to prepare plasmid DNA using the QIAprep 96 Turbo BioRobot kit (Qiagen). For sequencing of the ITS region cloned into the pGEM-T vector, the universal vector primers M13(-21) and M13rev were used on the plasmid preparation as target. Cloning resulted in the recognition of two distinct copies of the ITS, both with $<68 \%$ sequence similarity to M. xenopi. A $93 \%$ sequence similarity was noted between the two ITS copies. The presence of multiple copies of the 16S-23S ITS region, thus possibly multiple rRNA operons,

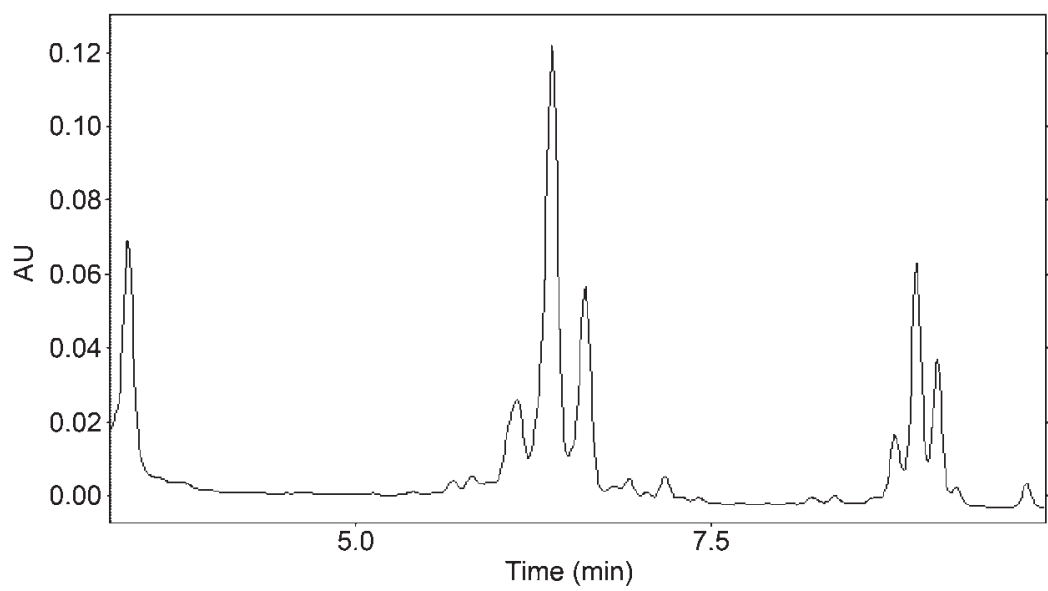

Fig. 1. Mycolic acid patterns of Mycobacterium noviomagense sp. nov. obtained by HPLC analysis. One early and one late cluster of peaks are present, similar to $M$. xenopi. The first (left) and last (right) peaks are the low and high weight molecular standards. 


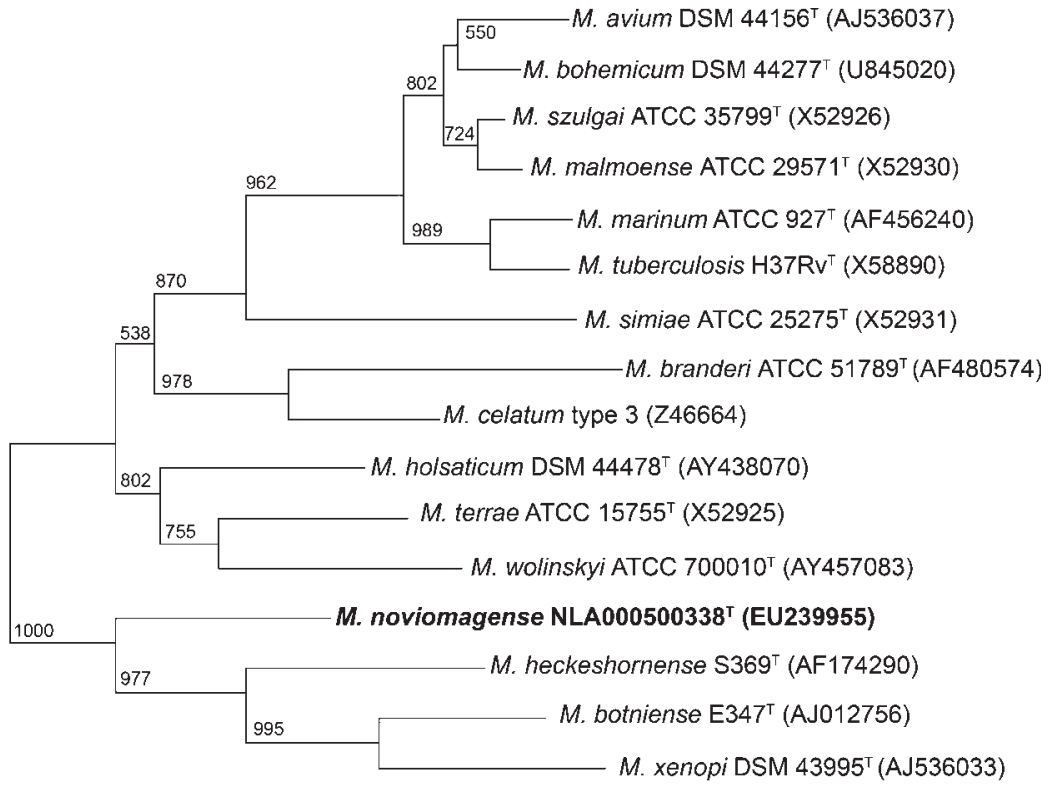

Fig. 2. Phylogenetic relationship of the type strain of the novel species, $M$. noviomagense sp. nov., and related species of the genus Mycobacterium, based on 16S rRNA gene sequences. The neighbour-joining tree was created and bootstrapped 1000 times with CLUSTAL_x (Thompson et al., 1997) and visualized with LOFT (Levels of Orthology through Phylogenetic Trees; van der Heijden et al., 2007). Bootstrap values are indicated at the nodes. is unexpected. This phenomenon has not been described for M. xenopi or for closely related slow-growing NTM species and thus supports the separate species status of the novel isolates.

The $r p o B$ gene sequence of the novel isolates was $95 \%$ similar to that of the recently described species Mycobacterium seoulense and only $92 \%$ similar to that of M. xenopi. For the hsp65 gene sequence, the most closely related sequences $(95 \%)$ were found among members of the Mycobacterium avium complex and Mycobacterium branderi, with $<93 \%$ similarity to $M$. xenopi. The considerable divergence in these two targets from the otherwise related cluster comprising $M$. xenopi, $M$. botniense and $M$. heckeshornense supports the separate species status of the novel isolates. The hsp65 and rpoB gene sequences were aligned with those of related mycobacterial species, as for the $16 \mathrm{~S}$ rRNA gene sequence. The resulting topologies and trees are available as Supplementary Figs S1 and S2 (in IJSEM Online).

The hsp65 gene PRA results for the novel isolates, M. xenopi, Mycobacterium tuberculosis $\mathrm{H}_{37 \mathrm{Rv}^{\mathrm{T}}}$ and M. botniense ATCC $700701^{\mathrm{T}}$ are shown in Fig. 3. For the novel isolates, digestion with BstEII resulted in fragments of $240 / 120 / 100 \mathrm{bp}$, digestion with HaeIII gave fragments of 130/10/70/45 bp. A PRAsite (http://app.chuv.ch/prasite/index.html) comparison showed this to be a unique fragment length combination. Isolates of $M$. xenopi and M. tuberculosis tested simultaneously were correctly identified using the PRAsite database; no entry was found for M. botniense.

\section{Description of Mycobacterium noviomagense sp. nov.}

Mycobacterium noviomagense (no.vi.o.ma.gen'se. N.L. neut. adj. pertaining to Noviomagus, the Roman name of
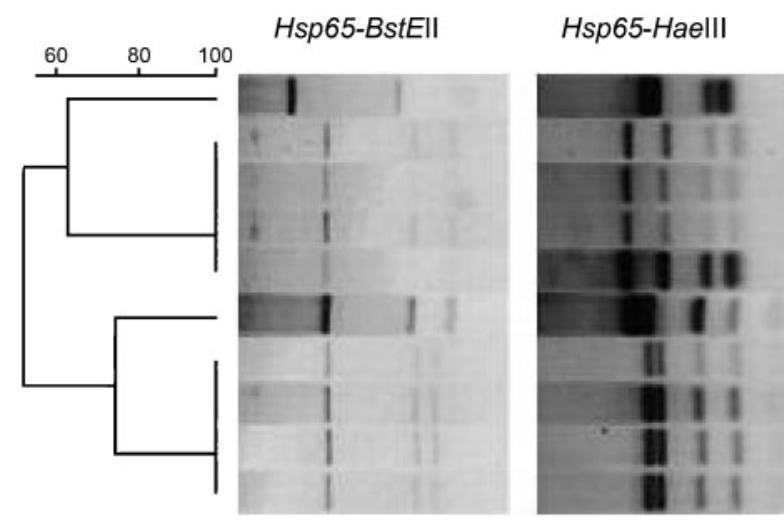

RIVM No.

Species

NLA000600819

NLA000200874

NLA000300775

NLA000300804

NLA000302134

NLA0000H37RV

NLA000300819

NLA000401111

NLA000401383

NLA000500338

\section{M. botniense \\ M. xenopi I \\ M. xenopi II \\ M. xenopi I \\ M. xenopi II}

M. tuberculosis

$M$. noviomagense

$M$. noviomagense

$M$. noviomagense

$M$. noviomagense

Fig. 3. PRA typing results for the hsp65 gene. Different fragment length patterns are observed for M. botniense (lane 1 ), $M$. xenopi (lanes 2-5), M. tuberculosis H37Rv ${ }^{\top}$ (lane 6) and strain NLA000500338 ${ }^{\top}$ (M. noviomagense sp. nov.; lanes 7-10). 
the major city in the endemic region in the Netherlands and the location of the reference hospital; current name: Nijmegen).

Acid-fast and Gram-positive rods. Colonies are nonchromogenic and appear after 4 weeks of culture at $37^{\circ} \mathrm{C}$, no growth occurs at $45{ }^{\circ} \mathrm{C}$. Negative in tests for urease, Tween 80 hydrolysis, niacin production, nitrate reductase, acid phosphatase and semi-quantitative catalase. Can be readily identified by its unique rRNA gene sequences.

The type strain, NLA000500338 $\left(=\mathrm{DSM} 45145^{\mathrm{T}}=\mathrm{CIP}\right.$ $\left.109766^{\mathrm{T}}\right)$, was recovered from sputum.

\section{Acknowledgements}

We respectfully thank Dr Pirjo Torkko of the Laboratory of Environmental Microbiology, National Public Health Institute, Kuopio, Finland, for providing us with M. botniense ATCC $700701^{\mathrm{T}}$ for comparative analysis. We thank Rebecca Millecamps at Innogenetics, Gent, Belgium, for assistance with the ITS sequencing and Anita Schuerch for assistance with the phylogenetic analyses.

\section{References}

Centers for Disease Control and Prevention (1996). Standardized Method for HPLC Identification of Mycobacteria. Atlanta, US: Department of Health and Human Services, Public Health Service.

Dauendorffer, J. N., Laurain, C., Weber, M. \& Dailloux, M. (2002). In vitro sensitivity of Mycobacterium xenopi to five antibiotics. Pathol Biol 50, 591-594.

Griffith, D. E., Aksamit, T., Brown-Elliot, B. A., Catanzaro, A., Daley, C., Gordin, F., Holland, S. M., Horsburgh, R., Huitt, G. \& other authors (2007). An official ATS/IDSA statement: diagnosis, treatment, and prevention of nontuberculous mycobacterial diseases. Am J Respir Crit Care Med 175, 367-416.

Hall, L., Doerr, A., Wohlfiel, S. L. \& Roberts, G. D. (2003). Evaluation of the MicroSeq system for identification of mycobacteria by $16 \mathrm{~S}$ ribosomal DNA sequencing and its integration into a routine mycobacteriology laboratory. J Clin Microbiol 41, 1447-1453.

Henry, M. T., Inamdar, L., O’Riordain, D., Schweiger, M. \& Watson, J. P. (2004). Nontuberculous mycobacteria in non-HIV patients: epidemiology, treatment and response. Eur Respir J 23, 741-746.

Kim, B. J., Lee, S. H., Lyu, M. A., Kim, S. J., Bai, G. H., Chae, G. T., Kim, E. C., Cha, C. Y. \& Kook, Y. H. (1999). Identification of mycobacterial species by comparative sequence analysis of the RNA polymerase gene (rpoB). J Clin Microbiol 37, 1714-1720.

Lévy-Frébault, V. V. \& Portaels, F. (1992). Proposed minimal standards for the genus Mycobacterium and for description of slowly growing Mycobacterium species. Int J Syst Bacteriol 42, 315-323.

Portaels, F. (1995). Epidemiology of mycobacterial diseases. Clin Dermatol 13, 207-222.
Roth, A., Fischer, M., Hamid, M. E., Michalke, S., Ludwig, W. \& Mauch, H. (1998). Differentiation of phylogenetically related slowly growing mycobacteria based on 16S-23S rRNA gene internal transcribed spacer sequences. J Clin Microbiol 36, 139-147.

Roth, A., Reischl, U., Schonfeld, N., Naumann, L., Emler, S., Fischer, M., Mauch, H., Loddenkemper, R. \& Kroppenstedt, R. M. (2000). Mycobacterium heckeshornense sp. nov., a new pathogenic slowly growing Mycobacterium sp. causing cavitary lung disease in an immunocompetent patient. J Clin Microbiol 38, 4102-4107.

Springer, B., Stockman, L., Teschner, K., Roberts, G. D. \& Bottger, E. C. (1996). Two-laboratory collaborative study on identification of mycobacteria: molecular versus phenotypic methods. J Clin Microbiol 34, 296-303.

Telenti, A., Marchesi, F., Balz, M., Bally, F., Bottger, E. C. \& Bodmer, T. (1993). Rapid identification of mycobacteria to the species level by polymerase chain reaction and restriction enzyme analysis. J Clin Microbiol 31, 175-178.

Thompson, J. D., Gibson, T. J., Plewniak, F., Jeanmougin, F. \& Higgins, D. G. (1997). The CLUSTAL_X windows interface: flexible strategies for multiple sequence alignment aided by quality analysis tools. Nucleic Acids Res 25, 4876-4882.

Torkko, P., Suomalainen, S., Livanainen, E., Suutari, M., Tortoli, E., Paulin, L. \& Katila, M.-L. (2000). Mycobacterium xenopi and related organisms isolated from stream waters in Finland and description of Mycobacterium botniense sp. nov. Int J Syst Evol Microbiol 50, 283289.

Tortoli, E. (2003). Impact of genotypic studies on mycobacterial taxonomy: the new mycobacteria of the 1990s. Clin Microbiol Rev 16, 319-354.

Tortoli, E., Bartolini, A., Böttger, E. C., Emler, S., Garzelli, C., Magliano, E., Mantella, A., Rastogi, N., Rindi, L. \& other authors (2001). Burden of unidentifiable mycobacteria in a reference laboratory. J Clin Microbiol 39, 4058-4065.

van Crevel, R., de Lange, W. C. M., Vanderpuye, N. A., van Soolingen, D., Hoogkamp-Korstanje, J. A. A., van Deuren, M., Kullberg, B. J., van Herwaarden, C. \& van der Meer, J. W. (2001). The impact of nontuberculous mycobacteria on management of presumed pulmonary tuberculosis. Infection 29, 59-63.

van der Heijden, R. T., Snel, B., van Noort, V. \& Huynen, M. A. (2007). Orthology prediction at scalable resolution by phylogenetic tree analysis. BMC Bioinformatics 8, 83.

van Ingen, J., Boeree, M. J., de Lange, W. C. M., Hoefsloot, W., Bendien, S. A., Magis-Escurra, C., Dekhuijzen, P. N. R. \& van Soolingen, D. (2008). Mycobacterium xenopi clinical relevance and determinants, the Netherlands. Emerg Infect Dis 14, 385-389.

van Klingeren, B. \& Pullen, W. (1993). Glutaraldehyde resistant mycobacteria from bronchoscope washers. J Hosp Infect 25, 147-149.

van Klingeren, B., Dessens-Kroon, M., van der Laan, T., Kremer, K. \& van Soolingen, D. (2007). Drug susceptibility testing of Mycobacterium tuberculosis complex using a high throughput, reproducible, absolute concentration method. J Clin Microbiol 45, 2662-2668. 